\title{
Relação entre percepção subjetiva do esforço e marcadores salivares em atletas de atletismo
}

http://dx.doi.org/10.11606/1807-5509201800020159

\author{
Bruno Pereira MELO* \\ Ramon CRUZ ${ }^{* *}$ \\ Francisco de Assis MANOEL* \\ Fernando Roberto de OLIVEIRA ${ }^{* * *}$ \\ Solange Marta Franzói de MORAES*
}

* Programa de Pósgraduação Associado em Educação Física, Universidade Estadual de Maringá, Maringá, PR, Brasil.

${ }^{* *}$ Programa de Pósgraduação Associado em Educação Física, Universidade Federal de Juiz de Fora, Juiz de Fora, MG, Brasil.

*** Universidade Federal de Lavras, Lavras, MG, Brasil.

\section{Resumo}

0 objetivo do estudo foi descrever a distribuição das Cargas de Treinamento (CT) pelo método da percepção subjetiva de esforço (PSE) da sessão e verificar a sua associação com as alterações de marcadores salivares (MS) em atletas de atletismo durante um camping de treinamento. Participaram do estudo, 44 atletas $(17,5 \pm 2,7$ anos; $175,1 \pm 9,0 \mathrm{~cm} ; 63,9 \pm 10,7 \mathrm{Kg})$ sendo, 11 velocistas, 11 fundistas, 11 saltadores e 11 arremessadores, avaliados durante oito sessões de treinamento (duração entre 120 e 150 minutos). Amostras de saliva foram colhidas antes e após o camping, avaliando as concentrações de cortisol, proteínas totais e osmolaridade. A PSE foi obtida trinta minutos após cada sessão, utilizando a escala CR10. A normalidade dos dados foi verificada pelo teste de Shapiro-Wilk. A comparação entre grupos foi realizada por ANOVA e a correlação entre PSE e MS foi obtida pela correlação de Pearson, adotando $p<0,05$. A distribuição das CT apresentaram características ondulatórias e alta variabilidade. Houve aumento na osmolaridade salivar nos velocistas $(55,0 \pm 14,0 \mathrm{vs}$. $68,7 \pm 22,6 \mathrm{m0sm} / \mathrm{L} ; \mathrm{p}=0,003)$ e saltadores $(60,9 \pm 17,9 \mathrm{vs} .77,8 \pm 18,7 \mathrm{m0sm} / \mathrm{L} ; \mathrm{p}=0,001)$. Os fundistas apresentaram maiores niveis de cortisol $(p=0,007)$ comparado aos demais grupos. Não foram encontradas correlações entre a PSE da sessão e MS. Sendo assim, a combinação de avaliações perceptuais e fisiológicas deve ser priorizada para um melhor controle e monitoramento de atletas na modalidade de atletismo.

Palavras-chave: Carga interna de treinamento; Cortisol; Treinamento físico; Desempenho atlético.

\section{Introdução}

O sucesso em competiçóes esportivas depende, em grande parte, de um elaborado programa de treinamento, o qual deve ser prescrito com cargas adequadas, com períodos de recuperação apropriados, visando uma otimizaçáo das adaptaçóes ao treinamento ${ }^{1,2}$. Avaliar e quantificar as cargas externas (distância percorrida, duração, velocidade) e cargas internas (alteraçôes fisiológicas) do treinamento, tem sido considerado um grande desafio para técnicos e treinadores de diferentes modalidades esportivas ${ }^{3-5}$, visto que a exposição do atleta a cargas excessivas, associada à monotonia das sessôes de treinos, podem gerar adaptaçóes negativas, levando à queda do desempenho que pode evoluir para o overtraining ${ }^{6-10}$.

Diversos métodos foram propostos para quantificar as cargas de treinamento, incluindo a utilização de questionários ${ }^{9}$, concentração de metabólitos (lactato) ${ }^{10}$, frequência cardíaca $(\mathrm{FC})^{11,12}$, consumo de oxigênio $\left(\mathrm{VO}_{2}\right)$ e Percepção Subjetiva de Esforço (PSE) ${ }^{13-16}$. No entanto, um conjunto maior de variáveis pode 
indicar com maior precisão o estresse imposto ao organismo do atleta durante o treinamento ${ }^{17-18}$. Neste sentido, a associação de variáveis fisiológicas, bioquímicas, imunológicas e perceptuais tornase uma estratégia atraente para avaliar a carga de treinamento ${ }^{2,19-20}$. Além disso, preconiza-se que estas avaliaçóes interfiram o mínimo possível no cotidiano dos atletas, portanto, ferramentas de fácil aplicação e de baixo custo sáo indicadas para o controle das cargas internas de treinamento.

Neste sentido, a medida de marcadores salivares é considerada uma estratégia não invasiva e de fácil aplicação para o monitoramento fisiológico em atletas de alto nível, uma vez que avaliaçóes de osmolaridade, proteínas totais e cortisol salivar têm sido amplamente utilizados para o monitoramento do nível de hidratação, estado nutricional e estresse fisiológico respectivamente ${ }^{21-23}$. Além disso, a PSE é considerada uma ferramenta de fácil acesso para a quantificação da carga de treinamento, uma vez que o método da PSE da sessão possibilita o monitoramento individual das sessões de treinos, permitindo uma comparação entre o que foi prescrito pelo treinador e o que foi percebido pelo atleta, minimizando assim as chances de ocorrência do overtraining ${ }^{2,4-7}$.

Devido às suas características (corridas, saltos e arremessos), o atletismo exige que treinadores tenham uma quantidade ótima de informaçóes para as prescriçóes, acompanhamento e avaliação das sessóes de treinamento. Neste sentido, a PSE da sessão é uma ferramenta importante para controle das cargas internas de treino ${ }^{13}$, e sua relação com as alteraçóes fisiológicas em atletas de atletismo de diferentes provas ainda não foi investigada. Logo, o entendimento sobre a associação entre estas variáveis pode ampliar as possibilidades nas tomadas de decisão para o treinamento esportivo.

Portanto, o objetivo do estudo foi verificar a distribuição das cargas de treinamento pelo método da PSE da sessão em atletas de atletismo de diferentes provas durante um camping de treinamento e verificar se este método se correlaciona com as possíveis alterações fisiológicas em marcadores salivares. A hipótese do estudo é que o método da PSE da sessão possui associação com alteraçóes fisiológicas de cortisol, proteínas totais e osmolaridade salivar independente da distribuição das cargas de treinamento e da característica de cada prova em atletas de atletismo.

\section{Métodos}

\section{Sujeitos}

O estudo foi realizado durante um camping de treinamento de jovens atletas brasileiros ranqueados pela Confederação Brasileira de Atletismo, classificados entre os melhores do país. A amostra foi constituída por 44 atletas de atletismo do sexo masculino, sendo 11 corredores de provas de velocidade, 11 corredores de provas de fundo, 11 saltadores e 11 arremessadores. As características dos atletas de cada grupo estão apresentadas na TABELA 1.

Para a composição dos grupos foram adotados os seguintes critérios: corredores de velocidade (velocistas atletas que competiam em provas com distância até 400 $\mathrm{m}$ rasos ou com barreiras), fundo (fundistas - atletas que competiam em provas de $3000 \mathrm{~m}$ rasos ou com obstáculo, $5000 \mathrm{~m}$ e $10000 \mathrm{~m}$ ), saltadores (atletas que competiam em provas de salto em altura, distância e triplo) e arremessadores (atletas que competiam em provas de arremesso de peso).

Os critérios de inclusão adotados foram: atletas com o mínimo de dois anos de treinamento, estar no ranking nacional da Confederação Brasileira de Atletismo, possuir uma frequência de treinamento de cinco vezes semanais e não possuir restrição física e/ou médica que impedisse a prática do treinamento físico.

Após a autorizaçáo e o consentimento dos técnicos, todos os atletas foram pré-informados sobre os riscos aos quais estariam sujeitos e assinaram um termo de consentimento livre e esclarecido aprovado pelo comitê de ética (CEP/ UFJF no. 945.274/2014) para participar do projeto respeitando as normas para pesquisa envolvendo seres humanos de acordo com a declaração de Helsink, de 1964.

\section{Procedimentos}

Todos os atletas passaram por uma avaliaçáo diagnóstica composta por anamnese, antropometria e coleta de saliva, em seguida, foram acompanhados durante oito sessóes de treinamento realizadas em quatros dias no período matutino (9h às $11 \mathrm{~h} 30) \mathrm{e}$ vespertino (15h às $17 \mathrm{~h} 30)$. 
Os treinamentos de cada grupo foram planejados e realizados pelo treinador específico de cada prova com duraçáo total de 120 a 150 minutos. Em todos os treinamentos foram seguidas a seguinte ordem: aquecimento $(10 \mathrm{~min})$, exercícios de flexibilidade (alongamentos ativo e passivo, 10-20 min), exercícios de coordenação motora (educativos, 20-30 min), parte principal (treinos físicos e/ou técnicos específico para cada grupo, 70-80 min) e recuperação $(10 \mathrm{~min}) \mathrm{em}$ cada sessão. Os treinamentos diferenciaram em cada grupo apenas na parte principal, sendo específico para a característica de prova de cada atleta em seu respectivo grupo. Todos os atletas se encontravam no período básico acumulativo da temporada de treinamentos.

O camping de treinamento foi realizado durante seis dias consecutivos envolvendo atletas de vários estados do país. Para garantir o mesmo intervalo de tempo entre a última sessão de treinamento e o horário das avaliaçóes, a coleta de saliva e a PSE da sessão foram obtidas a partir do segundo dia de treinamento, reservando o primeiro dia do camping para a seleção da amostra e familiarização com os protocolos de avaliação.

Todas as sessôes de treinamentos foram realizadas sempre no mesmo horário e local, assim como os intervalos de descanso. Durante a realização do camping foi fornecido aos atletas o mesmo cardápio para as refeiçôes do café da manhâ, almoço e jantar. O QUADRO 1 apresenta o delineamento experimental, assim como os horários das avaliaçóes, refeiçóes, treinamento e descanso dos atletas durante o camping de treinamento.

QUADRO 1 - Delineamento experimental: horário das avaliações, refeições, treinamento e descanso durante o camping de treinamento.

\begin{tabular}{|c|c|c|c|c|c|c|c|}
\hline & Horário & Dia 1 & Dia 2 & Dia 3 & Dia 4 & Dia 5 & Dia 6 \\
\hline & $7 \mathrm{~h}$ & Despertar & Despertar & Despertar & Despertar & Despertar & Despertar \\
\hline & $7 \mathrm{~h}-8 \mathrm{~h}$ & & $\begin{array}{l}\text { Coleta } \\
\text { Saliva }\end{array}$ & & & & Coleta Saliva \\
\hline & $8 \mathrm{~h}-8 \mathrm{~h} 30$ & $\begin{array}{l}\text { Café da } \\
\text { manhã }\end{array}$ & $\begin{array}{l}\text { Café da } \\
\text { manhã }\end{array}$ & $\begin{array}{l}\text { Café da } \\
\text { manhã }\end{array}$ & $\begin{array}{l}\text { Café da } \\
\text { manhã }\end{array}$ & $\begin{array}{l}\text { Café da } \\
\text { manhã }\end{array}$ & Café da manhã \\
\hline \multirow{7}{*}{ 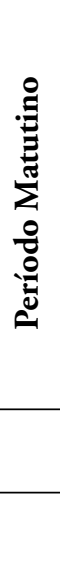 } & \multirow{5}{*}{$9 \mathrm{~h}-11 \mathrm{~h} 30$} & Divulgação & $\begin{array}{c}\text { Antro } \\
\text { pometria }\end{array}$ & & & & \multirow{5}{*}{$\begin{array}{c}\text { Encerramento } \\
\text { Camping }\end{array}$} \\
\hline & & Estudo & & & & & \\
\hline & & Treinamento & Treino 1 & Treino 3 & Treino 5 & Treino 7 & \\
\hline & & & $\begin{array}{l}\text { PSE da } \\
\text { Sessáo }\end{array}$ & $\begin{array}{l}\text { PSE da } \\
\text { Sessão }\end{array}$ & $\begin{array}{l}\text { PSE da } \\
\text { Sessão }\end{array}$ & $\begin{array}{l}\text { PSE da } \\
\text { Sessão }\end{array}$ & \\
\hline & & $\begin{array}{c}\text { Familiarização } \\
\text { da PSE }\end{array}$ & & & & & \\
\hline & $12 \mathrm{~h}-13 \mathrm{~h} 30$ & Almoço & Almoço & Almoço & Almoço & Almoço & \\
\hline & $13 \mathrm{~h} 30-15 \mathrm{~h}$ & Descanso & Descanso & Descanso & Descanso & Descanso & \\
\hline \multirow{3}{*}{ 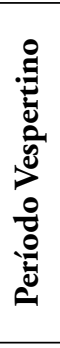 } & \multirow{3}{*}{$15 \mathrm{~h}-17 \mathrm{~h} 30$} & $\begin{array}{l}\text { Anamnese/ } \\
\text { Separação dos } \\
\text { grupos }\end{array}$ & Treino 2 & Treino 4 & Treino 6 & Treino 8 & \\
\hline & & Treinamento & $\begin{array}{l}\text { PSE da } \\
\text { Sessão }\end{array}$ & $\begin{array}{l}\text { PSE da } \\
\text { Sessão }\end{array}$ & $\begin{array}{l}\text { PSE da } \\
\text { Sessáo }\end{array}$ & $\begin{array}{l}\text { PSE da } \\
\text { Sessão }\end{array}$ & \\
\hline & & $\begin{array}{c}\text { Familiarização } \\
\text { da PSE }\end{array}$ & & & & & \\
\hline & $18 \mathrm{~h}-19 \mathrm{~h} 30$ & Jantar & Jantar & Jantar & Jantar & Jantar & \\
\hline & $22 \mathrm{~h}$ & Repouso & Repouso & Repouso & Repouso & Repouso & \\
\hline
\end{tabular}




\section{Quantificaçáo das Cargas de Treinamento}

A determinação e a quantificação das cargas de treinamento foram realizadas pelo método da PSE da sessáo ${ }^{15}$. Trinta minutos após o término da sessão de treinamento o atleta respondeu a seguinte pergunta: "Como foi a sua sessão de treino?" A resposta ao questionamento foi fornecida a partir da escala CR10 de Borg (1982) adaptada por Foster et al. ${ }^{15}$.

O cálculo da carga de treinamento consistiu na multiplicação do escore da PSE pela duração total da sessão (em minutos) do treinamento, incluindo aquecimento, parte principal e recuperação, correspondendo assim a um valor expresso em unidades arbitrárias (UA). Além da magnitude das cargas de treino, a monotonia do treinamento em cada atleta foi calculada pela média das cargas semanais dividida pelo desvio padrão ${ }^{13}$. O nível de esforço do treinamento em cada grupo foi obtido pelo cálculo de training strain, na qual foi dada pela multiplicação da monotonia pelo somatório das cargas acumuladas durante a semana de treinamento ${ }^{15}$.

\section{Marcadores Salivares}

Amostras de saliva foram colhidas de cada atleta de forma estimulada durante um minuto, sob jejum de oito horas, no período da manhã (entre 7h30-8h00), sendo realizada em dois momentos: pré e pós-oito sessóes de treinamento, através de tubos próprios contendo rolos de algodão em seu interior (Salivette ${ }^{\oplus}$, SARSTEDT). Os atletas foram instruídos a não escovar os dentes e realizar um enxague bucal com água previamente à coleta da saliva.

As amostras de saliva foram centrifugadas a $3000 \mathrm{rpm}$ durante 15 minutos e armazenadas em freezer $\mathrm{a}-80^{\circ} \mathrm{C}$ para posteriormente serem analisadas as concentraçóes de cortisol utilizando o teste enzimático de ELISA (Kit Diametra, Itália Ref. DKO 020), respeitando todas as instruçôes do fabricante. A osmolaridade salivar foi avaliada através de Osmômetro VAPRO ${ }^{\circledR}$ (Wescor 5520) e a concentração de proteínas totais identificadas pelo método de BRADFORD ${ }^{24}$.

\section{Análise estatística}

A caracterização da amostra foi feita a partir da estatística descritiva (média e desvio padrão). Os pressupostos paramétricos de normalidade e de igualdade de variâncias foram avaliados pelo teste Shapiro-Wilk e pelo teste de Levene, respectivamente. A partir dos resultados, para a comparação entre os quatros grupos (velocistas, fundistas, saltadores e arremessadores), foi utilizada a Análise de Variância (ANOVA), um fator para dados com distribuição normal e o teste de Kruskal Wallis para dados náo paramétricos. Quando adicionalmente à comparação entre grupos foi também empregada a comparação entre momentos (pré versus pós), fez-se uso da ANOVA mista de medidas repetidas. Quando observada a diferença na ANOVA mista de medidas repetidas, foi empregado o post-hoc de Bonferroni.

As correlaçóes entre o percentual de variação das variáveis fisiológicas com a monotonia de treino, strain e a média das cargas de treinamento ao longo da semana foram realizadas por meio do coeficiente de correlação de Pearson. A análise qualitativa das correlaçôes foram realizadas a partir dos pontos de corte estabelecidos por Hopkins et al..$^{25}$ : trivial $(<0,01)$, pequena (entre 0,1 e 0,29$)$, moderada (entre 0,3 e 0,49 ), elevada (entre 0,5 e 0,69 ), muito elevada (entre 0,7 e 0,89 ) e quase perfeita (entre 0,9 e 1 ). Todas as análises foram feitas no software SPSS (v.20, SPSS Inc., Chicago, IL, USA), sendo adotado o nível de significância de 5,0\% $(\mathrm{p}<0,05)$.

\section{Resultados}

Não foram encontradas diferenças significativas na idade, IMC e tempo de treinamento dos atletas quando comparados entre os grupos. Os resultados estáo apresentados na TABELA 1.

A distribuição das cargas de treinamento pelo método da PSE da sessão não apresentou diferenças significantes quando comparadas entre os grupos (FIGURA 1). A monotonia e a medida de training strain também não apresentaram diferença significante, demostrando que independente da especificidade da prova de cada atleta a característica geral das cargas internas de treinamento foram similares para este período da temporada esportiva (TABELA 2). 
TABELA 1 - Descrição e comparação da idade, índice de massa corporal (IMC), tempo de treinamento e nível de competição oficial já disputada em cada grupo.

\begin{tabular}{lccccc}
\hline & Velocistas & Fundistas & Saltadores & Arremessadores & P \\
\hline Número & 11 & 11 & 11 & 11 & - \\
Idade (anos) & $17 \pm 2,7$ & $18 \pm 3,4$ & $18 \pm 2,4$ & $17 \pm 2,3$ & 0,73 \\
IMC (kg/m2) & $20,6 \pm 2,4$ & $19,3 \pm 1,9$ & $20,3 \pm 1,7$ & $22,6 \pm 2,9$ & 0,94 \\
Tempo treinamento (anos) & $4 \pm 1,0$ & $4 \pm 1,1$ & $4 \pm 1,4$ & $4 \pm 1,2$ & - \\
Nível estadual, n (\%) & $1(9,1 \%)$ & $2(18,2 \%)$ & $2(18,2 \%)$ & $4(36,4 \%)$ & \\
Nível nacional, n (\%) & $8(72,7 \%)$ & $9(81,8 \%)$ & $8(72,7 \%)$ & $7(63,6 \%)$ & - \\
Nível internacional, n (\%) & $2(18,2 \%)$ & - & $1(9,1 \%)$ & - \\
\hline
\end{tabular}

TABELA 2 - Nível de monotonia e strain identificados em cada grupo de atletas durante o camping de treinamento.

\begin{tabular}{lccccc}
\hline & $\begin{array}{c}\text { Velocistas } \\
(\mathbf{n = 1 1})\end{array}$ & $\begin{array}{c}\text { Fundistas } \\
(\mathbf{n = 1 1})\end{array}$ & $\begin{array}{c}\text { Saltadores } \\
(\mathbf{n = 1 1})\end{array}$ & $\begin{array}{c}\text { Arremessadores } \\
(\mathbf{n = 1 1})\end{array}$ & $\mathbf{P}$ \\
\hline $\begin{array}{l}\text { Monotonia (UA) } \\
\begin{array}{l}\text { Training strain } \\
\text { (UA) }\end{array}\end{array}$ & $2,1 \pm 0,6$ & $1,9 \pm 0,4$ & $2,0 \pm 0,6$ & $2,2 \pm 0,6$ & 0,81 \\
\hline
\end{tabular}

PSE = Percepção Subjetiva de Esforço; $U A=$ Unidades Arbitrárias; $\mathrm{P}=$ significância.

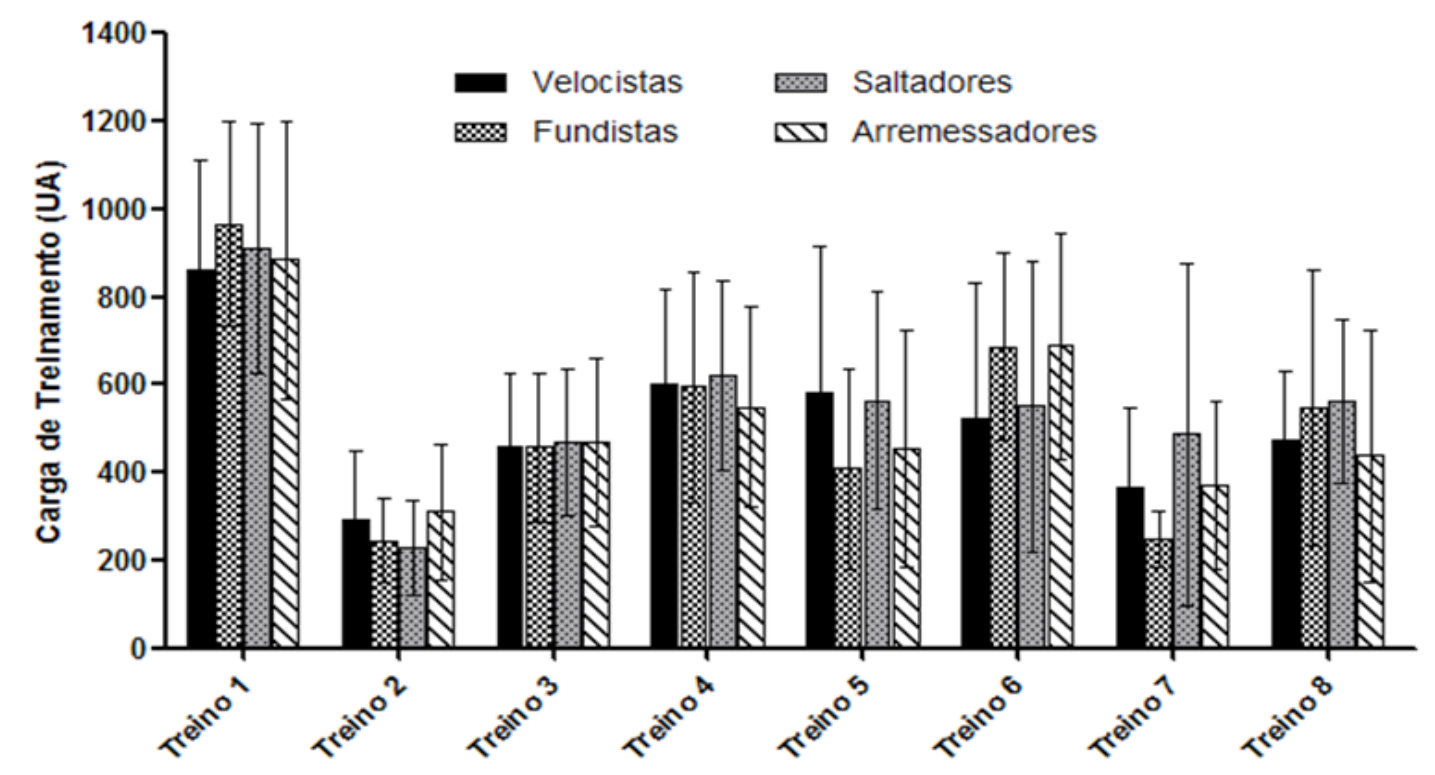

FIGURA 1 - Comparação e descrição das cargas de treino pelo método da PSE da sessão com as características de prova de cada atleta. 
t: Valores de referência de acordo com WaLSH et al. ${ }^{34}$, *: Diferença significante comparando o momento pré vs. pós em cada grupo; \#: Diferença significante em relação aos grupos velocistas, saltadores e arremessadores.
Análise qualitativa do Coeficiente de Correlação de Pearson de acordo com HopkINs et al. ${ }^{25}$ : trivia $(<0,01)$, pequena (entre 0,1 e 0,29) moderada (entre $0,3 \mathrm{e}$ 0,49 ), elevada (entre 0,5 e 0,69 ), muito elevada (entre $0,7 \mathrm{e}$ $0,89)$ e quase perfeita (entre 0,9 e 1); UA: Unidade arbitrária; PSE: Percepção Subjetiva de Esforço.
As análises de osmolaridade, proteínas totais e cortisol salivar estão apresentados na TABELA 3. Os resultados anteriores às sessóes de treinamento de osmolaridade, proteínas totais e cortisol salivar quando comparados os grupos não apresentaram diferenças significantes $(p>0,05)$. Ao analisar os valores de cortisol salivar após as sessóes de treinamento, os fundistas apresentaram valores maiores $(p=0,007)$ quando comparados aos demais atletas, indicando alguma relação nas respostas bioquímicas de acordo com o grupo de provas do atletismo. Em relação aos valores pós de osmolaridade e proteínas totais salivares não foram encontradas diferenças significantes entre os grupos $(\mathrm{p}>0,05)$.

Quando comparados às alteraçóes fisiológicas em cada grupo, os velocistas e os saltadores apresentaram um aumento significante $(\mathrm{p}=0,003 ; \mathrm{p}=0,001)$ na osmolaridade salivar e uma diminuiçãoo $(\mathrm{p}=0,013$; $\mathrm{p}=0,044)$ nos valores de cortisol salivar após o camping de treinamento. Em relação à concentração de proteínas totais não foram encontradas diferenças significantes em nenhum grupo $(\mathrm{p}>0,05)$.

Ao analisar o efeito principal nos marcadores salivares, para a osmolaridade foram encontrados efeitos do momento $(\mathrm{p}<0,001)$ e interação entre grupos e momentos $(p=0,016)$. O cortisol salivar apresentou efeito apenas na interaçáo entre grupos e momentos ( $\mathrm{p}=0,028)$. Os valores de proteínas totais náo apresentaram nenhum efeito principal.

De acordo com os pontos de corte estabelecidos por Hopkins et al..$^{25}$ foram encontradas correlaçóes pequenas (entre 0,1 e 0,29) e moderadas (entre 0,3 e 0,49$)$ entre o método da PSE da sessão (cargas de treinos, monotonia e strain) com o percentual de variação dos marcadores salivares (cortisol, proteínas totais e osmolaridade).

TABELA 3 - Comparação dos marcadores salivares de osmolaridade, proteínas totais e cortisol antes e após as oito sessões de treinamentos.

\begin{tabular}{|c|c|c|c|c|c|}
\hline & $\begin{array}{c}\text { Velocistas } \\
(n=11)\end{array}$ & $\begin{array}{c}\text { Fundistas } \\
(n=11)\end{array}$ & $\begin{array}{c}\text { Saltadores } \\
(\mathbf{n}=11)\end{array}$ & $\begin{array}{l}\text { Arremessadores } \\
(n=11)\end{array}$ & $\begin{array}{c}\text { Valor Referên- } \\
\text { cia } \dagger\end{array}$ \\
\hline \multicolumn{6}{|c|}{ Osmolaridade (mOsm/L) } \\
\hline Pré & $55,0 \pm 14,0$ & $62,9 \pm 12,5$ & $60,9 \pm 17,9$ & $52,1 \pm 10,4$ & \multirow{2}{*}{$41-61$} \\
\hline Pós & $68,8 \pm 22,6^{*}$ & $57,9 \pm 14,9$ & $77,8 \pm 18,7^{*}$ & $66,5 \pm 16,0$ & \\
\hline \multicolumn{6}{|c|}{ Proteínas totais $(\mathrm{mg} / \mathrm{mL})$} \\
\hline Pré & $0,4 \pm 0,2$ & $0,4 \pm 0,1$ & $0,4 \pm 0,2$ & $0,4 \pm 0,2$ & \multirow{2}{*}{$0,5-0,9$} \\
\hline Pós & $0,4 \pm 0,3$ & $0,4 \pm 0,3$ & $0,6 \pm 0,4$ & $0,5 \pm 0,2$ & \\
\hline \multicolumn{6}{|c|}{ Cortisol (ng/mL) } \\
\hline Pré & $3,2 \pm 1,4$ & $2,9 \pm 1,9$ & $2,7 \pm 1,1$ & $3,5 \pm 1,5$ & \multirow{2}{*}{$3-10$} \\
\hline Pós & $1,9 \pm 0,7^{*}$ & $3,9 \pm 2,3 \#$ & $2,15 \pm 1,1^{*}$ & $2,2 \pm 0,9$ & \\
\hline
\end{tabular}

TABELA 4 - Correlação entre o método da PSE da sessão e o percentual de variação de osmolaridade, proteínas totais e cortisol salivar em cada grupo.

\begin{tabular}{lccc}
\hline & \% Variaçáo Osmolaridade & \% Variaçáo Proteínas totais & $\begin{array}{c}\text { \% Variaçáo } \\
\text { Cortisol }\end{array}$ \\
\hline Velocistas & & & \\
Média PSE da sessão (UA) & $-0,22$ & 0,34 & $-0,20$ \\
Média carga de treino (UA) & $-0,14$ & 0,38 & $-0,27$ \\
Monotonia & $-0,21$ & 0,48 & 0,13 \\
$\quad$ Strain & $-0,15$ & 0,49 & $-0,12$ \\
\hline Fundistas & & & \\
Média PSE da sessão (UA) & $-0,26$ & $-0,54$ & $-0,33$ \\
Média carga de treino (UA) & $-0,28$ & $-0,51$ & $-0,24$ \\
Monotonia & $-0,52$ & $-0,35$ & 0,17 \\
$\quad$ Strain & $-0,49$ & $-0,50$ & $-0,29$ \\
\hline Saltadores & & & 0,49 \\
$\quad$ Média PSE da sessão (UA) & 0,10 & 0,17 & \\
\hline
\end{tabular}


TABELA 4 - Correlação entre o método da PSE da sessão e o percentual de variação de osmolaridade, proteínas totais e cortisol salivar em cada grupo.

\begin{tabular}{lccc}
\hline & \% Variaçáo Osmolaridade & \% Variaçáo Proteínas totais & $\begin{array}{c}\text { \% Variaçáo } \\
\text { Cortisol }\end{array}$ \\
\hline Média carga de treino (UA) & 0,12 & $-0,16$ & 0,46 \\
Monotonia & $-0,15$ & 0,20 & 0,23 \\
Strain & $-0,19$ & 0,27 & 0,35 \\
\hline Arremessadores & & & \\
Média PSE da sessão (UA) & $-0,51$ & $-0,46$ & 0,36 \\
Média carga de treino (UA) & $-0,32$ & $-0,44$ & 0,18 \\
Monotonia & $-0,22$ & 0,27 & 0,49 \\
Strain & $-0,35$ & $-0,17$ & $-0,48$ \\
\hline
\end{tabular}

\section{Discussão}

O objetivo do estudo foi verificar a distribuição das cargas de treinamento pelo método da PSE da sessão em atletas de atletismo de diferentes provas, verificando se este método se correlaciona com as possíveis alterações fisiológicas em marcadores salivares. O principal resultado deste estudo foi que a distribuição das cargas de treinos foi semelhante entre os grupos (velocistas, fundistas, saltadores e arremessadores) durante o período básico acumulativo da temporada. Além disso, as correlaçôes entre método da PSE da sessão e alterações fisiológicas de osmolaridade, proteínas totais e cortisol salivar foram pequenas e moderadas.

A distribuição semelhante das cargas de treinamento entre os grupos pode ser explicada pelo fato de que o camping possui como principal objetivo a interação entre técnicos, treinadores e atletas de diferentes equipes do país. Diante disso, as sessóes de treinamentos são compostas por exercícios cuja finalidade é promover a troca de experiências entre atletas e treinadores, enfatizando não apenas as especificidades de cada prova, mas sim, a ampla variedade de exercícios gerais para o atletismo. Além disso, por apresentar duas sessóes diárias de treinamento, o camping possui um alto volume de treinamentos que são distribuídos em sessôes com cargas intensas, moderadas e regenerativas provocando assim uma alta variabilidade na intensidade entre as sessôes de treinamento para todos os grupos. Durante um camping de treinamento de judocas, Viveiros et al. ${ }^{26}$ encontraram a mesma situação reportada no presente estudo.

Outro fator importante é que o camping de treinamento é realizado fora do período competitivo, assim, as intensidades das cargas de treinos neste período diferem das cargas regularmente realizadas no período competitivo ${ }^{26}$, fazendo com que técnicos e treinadores tenham um maior cuidado na prescrição e na distribuição das cargas de treinos. Portanto, o uso de ferramentas de fácil acesso e aplicabilidade para o controle das sessóes de treinamento e monitoramento fisiológico destes atletas é essencial para o período básico preparatório e ao longo de toda a temporada.

Neste sentido, a monotonia e training strain são parâmetros que podem ser utilizados para controle e monitoramento do treinamento ${ }^{13}$. Os resultados encontrados do presente estudo apontam maiores valores de training strain quando comparada a outras modalidades esportivas ${ }^{18,27}$. Isso pode ser explicado pelo fato que o training strain é influenciado diretamente pelo volume de treinamento. Deste modo, o camping foi realizado em um período específico da temporada caracterizado por um alto volume de treinos (básico preparatório), o que pode ter contribuído diretamente para estes achados.

De acordo com Nakamura et $\mathrm{al}^{2}{ }^{2}$, a magnitude e a monotonia das cargas de treinos podem influenciar respostas adaptativas ao treinamento, uma vez que a baixa variabilidade entre as sessóes associada ao elevado nível de monotonia apresentam alta correlação com a incidência de infecçóes do trato respiratório superior e adaptaçóes negativas, potencializando assim a chance de overtraining ${ }^{13}$. Portanto, o controle e o planejamento das cargas de treinos especialmente no período básico acumulativo são necessários para evitar adaptações indesejáveis ao treinamento.

Ao analisar os resultados pré dos marcadores salivares, foi verificado que não houve diferença significante entre os grupos, demonstrando que, para estas análises, os grupos partem de condiçóes 
fisiológicas semelhantes. Entretanto, os resultados obtidos após o camping de treinamento demonstraram que os velocistas e saltadores obtiveram um aumento significante da osmolaridade salivar.

A osmolaridade salivar é considerado um marcador potencialmente útil para analisar o estado de hidratação e recuperação de atletas ${ }^{21,28-29}$, possuindo alta correlação com a osmolaridade sérica e urinária ${ }^{30}$. $\mathrm{O}$ aumento da osmolaridade é encontrado em quadros de desidratação, sugerindo um mecanismo de transporte de água pura, de forma similar como ocorre à desidratação ou diluição da urina nos rins ${ }^{31}$.

O aumento na osmolaridade salivar encontrados nos velocistas e saltadores podem ser explicados pelo comportamento dos atletas em relaçáo à hidratação voluntária durante cada sessão de treinamento, uma vez que, altas taxas de ingestão de líquidos podem levar desconfortos abdominais possivelmente devido ao acúmulo de líquidos não absorvidos no cólon ou intestino delgado, levando à queda do rendimento durante o treinamento ${ }^{32}$. Além disso, a ação de osmorreceptores importantes na regulação e secreção de alguns hormônios, tais como a vasopressina, que regulam a percepção da sede e a tonicidade dos líquidos corporais, pode ser influenciada pelo treinamento físico, induzindo assim uma menor percepção da sede em atletas durante o treinamento ${ }^{33}$.

No presente estudo não foram encontradas diferenças significantes nas concentraçóes de proteínas totais após o camping de treinamento. Estes achados podem ser explicados pelo fato de que durante o camping de treinamento foi fornecido aos atletas o mesmo cardápio em todas as refeiçóes diárias, sendo controlado o horário de cada refeição. Outro fator importante que pode ter contribuído para estes resultados é a semelhança encontrada na distribuição das cargas de treinamentos e o tempo dos intervalos de descanso para todos os grupos, uma vez que o balanço entre o desgaste físico e a recuperação pode interferir diretamente no metabolismo basal dos atletas influenciando também a concentração de proteínas totais salivares.

As proteínas são os elementos mais abundantes e importantes da saliva, pois fornecem informações importantes para o monitoramento de atletas, uma vez que concentraçôes baixas de proteínas (hipoproteinemia) podem indicar má alimentação e, consequentemente, uma desnutrição proteica, fatores que podem afetar diretamente o rendimento e a recuperação de cada atleta ${ }^{22,27,34}$. Além disso, as proteínas desempenham um papel importante na homeostase fisiológica principalmente por contribuir na formação de enzimas, anticorpos, hormônios, contribuindo também no transporte de substâncias e na atividade muscular ${ }^{22}$. Neste sentido, a medida de proteínas totais salivares pode ser considerada uma avaliação importante para o monitoramento do estado nutricional de atletas de atletismo de diferentes provas especificamente no período básico da temporada.

Ao analisar os dados de cortisol após o camping (TABELA 3) foi verificado que os velocistas e saltadores apresentaram uma diminuição significante ( $p=0,013 ; p=0,044)$ nas concentraçóes de cortisol salivar. Este achado pode ser explicado pelo fato de que, quando se tem uma reserva adequada de glicogênio muscular, o cortisol pode ser inibido pela ação de outros hormônios, tais como o hormônio do crescimento e testosterona ${ }^{22}$ que são sintetizados em grande escala nos treinamentos de potência e força muscular ${ }^{23}$, que são treinos característicos desse grupo de atletas.

Ao comparar os resultados encontrados entre os grupos foi observado que os fundistas apresentaram maiores valores $(p=0,007)$ de cortisol salivar quando comparado aos demais após o camping de treinamento. Estes achados podem ser explicados pelo fato de que o cortisol é produzido em maior quantidade em treinos aeróbios prolongados, principalmente para estimular o metabolismo de lipídios e proteínas ${ }^{35,36}$. Nesse sentido, a combinação de exercícios aeróbios prolongados e exercícios intervalados de alta intensidade, são frequentemente realizados por corredores de fundo, o que pode ter contribuído para o aumento do cortisol em relação aos demais grupos ${ }^{36-37}$. Recentemente BalsalobreFERNÁNDEZ et al. ${ }^{37}$ encontraram uma associação entre o volume de treinamento (quilômetros percorridos) e a concentração de cortisol salivar em corredores de média e longa duração, demonstrando que, quanto maior o volume de treinamento semanal, maior a concentração de cortisol salivar. Sugerindo assim que corredores de média e longa duração possuem maior estresse fisiológico durante o período acumulativo de treinamento.

Outro ponto importante que deve ser levado em consideração é o efeito da última sessão de treinamento sobre as concentraçóes de cortisol. Estudos realizados por Tremblay, Copeland e VAN Helder ${ }^{38}$ demonstraram que as concentraçóes de cortisol podem estar elevadas mesmo após 24 horas da última sessão de treinamento. Apesar das avaliaçóes serem realizadas sob o mesmo intervalo 
de tempo em relação à última sessão, os fundistas possuem como característica principal uma maior quantidade de treinamentos físicos, enquanto que nos demais atletas são enfatizados treinos técnicos, o que pode ter contribuído para estes resultados.

As correlaçóes entre os marcadores salivares com a média das cargas internas de treinamento, monotonia e strain, apresentaram correlaçóes pequenas e moderadas. Estes resultados podem ser elucidados pelo fato de que a média das cargas de treinamentos, monotonia e strain são parâmetros acumulativos de todas as sessōes de treinamento. Entretanto, a osmolaridade, proteínas totais e cortisol salivar, podem sofrer alteraçóes do momento da coleta e não apenas do período acumulativo de todas as sessóes de treinamento. Além disso, vale ressaltar que as oscilaçóes na distribuiçáo da carga de treinamento encontradas durante o camping podem ter contribuído para uma maior adaptação ao treinamento e, consequentemente, uma diminuição de estresse fisiológico.

A associação entre a PSE da sessão e alterações fisiológicas já foram reportadas em outros estudos nas quais encontraram uma forte relação entre a PSE da sessão, consumo de oxigênio e frequência cardíaca ${ }^{39,40}$. No entanto, vale ressaltar que o presente estudo até o momento é o primeiro a verificar a relação entre os marcadores salivares e a PSE da sessão em atletas de atletismo de diferentes provas.
Por fim, algumas limitaçóes do presente estudo podem ser apontadas como: o fato de nâo realizar coleta e análises dos marcadores salivares ao final de cada sessão de treinamento e também a aplicação de um parâmetro para o monitoramento da recuperação física geral dos atletas. Estas análises adicionais possibilitariam verificar as alteraçóes fisiológicas em cada sessão de treinamento e também a recuperação física de cada atleta entre as sessóes. No entanto, essas análises são consideradas como complementares, o que não influenciou o principal objetivo deste estudo. Sendo assim, estudos posteriores podem considerar estas variáveis e também em períodos distintos da temporada esportiva.

Conclui-se que, durante o período básico da temporada, a distribuição das cargas de treinamentos foi semelhante para todos os atletas de atletismo independente das características das provas. No entanto, os fundistas apresentaram um maior estresse fisiológico quando comparado aos demais grupos. Neste sentido, sugere-se que durante esse período da temporada o controle das sessóes de treinamento seja realizado através de avaliaçóes perceptuais como a PSE da sessão e também por avaliaçóes fisiológicas por meio de marcadores salivares, pois essas são ferramentas de baixo custo e de fácil acesso e aplicabilidade que interferem o mínimo possível na rotina de treinamento dos atletas.

\section{Abstract}

\section{Relationship between of rating of perceived exertion and salivary markers in athletics athletes}

The aim of the study was to describe the distribution of training loads (TL) by the method of Subjective Rating of Perceived Exertion (RPE) sessions and verify your association with the changes of salivary markers (SM) in track and field athletes during a training. Participated in the study 44 athletes $(17.5 \pm 2.7$ years; $175.1 \pm 9.0 \mathrm{~cm}-1 ; 63.9 \pm 10.7 \mathrm{Kg}-1$ ) being 11 sprinters, 11 jumpers, 11 runners and 11 athletes shot puts, evaluated for eight training sessions (duration between 120-150 min-1). Saliva samples were collected before and after the camping to evaluate cortisol levels, osmolality and total protein. The RPE was obtained thirty minutes after each session using the CR10 scale. The data normality was verified by the Shapiro-Wilk test. The comparison between groups was performed by ANOVA one-way and correlation between RPE and SM were obtained by Pearson correlation, considering $p<0.05$. The distribution of TL they presented characteristic undulated and variability high. There was an increase in salivary osmolality in sprinters (55.0 \pm 14.0 vs. $68.7 \pm 22.6 \mathrm{mOsm} . \mathrm{L}-1 ; p=0.003)$ and jumpers $(60.9 \pm 17.9$ vs. $77.8 \pm 18.7 \mathrm{mOsm} . \mathrm{L}-1 ; p=0.001)$. The runners presented higher cortisol levels $(p=0.007)$ compared to other groups. There were no correlations between sessions RPE and SM. Thus, the combination of perceptual and physiological assessments should be prioritized for better control and monitoring in athletes athletic.

KEYWORDS: Internal load training; Cortisol; Physical training; Athletic performance. 


\section{Referências}

1. Kiviniemi AM, Hautala AJ, Kinnunen H, Tulppo MP. Endurance training guided individually by daily heart rate variability measurements. Eur J Appl Physiol. 2007;101(6):743-51.

2. Nakamura FY, Moreira A, Aoki MS. Monitoramento da carga de treinamento: a percepção subjetiva do esforço da sessão é um método confiável? Rev Edu Física UEM. 2010;21(1):1-11.

3. Baker D, Wilson G, Carlyon, R. Periodization: the effects of manipulating volume and intensity. J Strength Cond Res. $1994 ; 8(4): 235-42$.

4. Borresen J, Lambert MI. The quantification of training load, the training response and the effect on performance. Sports Med. 2009;39(9):779-95.

5. Eloumi M, Makni E, Moalla W, Bouaziz T, Tabka Z, Lac G, et al. Monitoring training load and fatigue in rugby sevens players. Asian J Sports Med. 2012;3(3):175-84.

6. Burini FHP, Oliveira EP, Burini RC. (Mal) adaptações metabólicas ao treinamento contínuo: concepções não consensuais de terminologia e diagnóstico. Rev Bras Med Esporte. 2010;16(5):388-92.

7. Costa LOP, Samulski DM. Overtraining em atletas de alto nível: uma revisão literária. Rev Bras Ciencia Mov. 2005;13(2):123-34.

8. Halson SL, Jeukendrup AE. Does overtraining exist? An analysis of overreaching and overtraining research. Sports Med. 2004;34(14):967-81.

9. Borresen J, Lambert MI. Validity of self-reported training duration. Int J Sports Sci Coach. 2006;1(4):353-9.

10. Swart J, Jennings CL. Use of blood lactate concentration as a marker of training status. South Afri J Sports Med. 2004;16(3):3-7.

11. Earnest CP, Jurca R, Church TS, Chicharro JL, Hoyos J, Lucia, A. Relation between physical exertion and heart rate variability characteristics in professional cyclists during the Tour of Spain. Bri J Sports Med. 2004;38(5):568-75.

12. Lucia A, Hoyos J, Santalla A, Earnest C, Chicharro JL. Tour de France versus Vuelta a España: which is harder? Med Sci Sports Exerc. 2003;35(5):872-8.

13. Foster C. Monitoring training in athletes with reference to overtraining syndrome. Med Sci Sports Exerc. 1998;30(7):1164-8.

14. Foster C, Daines E, Hector L, Snyder AC, Welsh R. Athletic performance in relation to training load. Wis Med J. 1996;95(6):370-4.

15. Foster C, Florhaug JA, Franklin J, Gottschall L, Hrovatin LA, Parker S, et al. A new approach to monitoring exercise training. J Strength Cond Res. 2001;15(1):109-15.

16. Foster C, Hector LL, Welsh R, Schrager M, Green MA, Snyder A. Effects of specific versus cross-training on running performance. Eur J App Physiol Occup Physiol. 1995;70(4):367-72.

17. Coutts AJ, Wallace LK, Slattery KM. Monitoring changes in performance, physiology, biochemistry, and psychology during overreaching and recovery in triathletes. Int J Sports Med. 2007;28(2):125-34.

18. Freitas VH. Monitoramento da carga de treinamento e marcadores de sobrecarga no voleibol. Juiz de Fora. Dissertação [Mestrado em Educação Física] - Universidade Federal de Juiz de Fora; 2012 [cited 2018 Feb 28]. Available from: http:// www.uff.br/pgedufisica/files/2017/09/disserta\%C3\%A7\%C3\%A3o-Final-Victor-Hugo.pdf

19. Cunha GS, Ribeiro JL, Oliveira AR. Sobretreinamento: teorias, diagnóstico e marcadores. Rev Bras Med Esporte. 2006;12(5):297-302.

20. Meeusen R, Duclos M, Gleeson M, Rietjens G, Steinacker J, Urhausen A. Prevention, diagnosis and treatment of the Overtraining Syndrome. Eur J Sports Sci. 2006;6(1):1-14.

21. Ely BR, Cheuvront SN, Kenefick RW, Sawka MN. Limitations of salivary osmolality as a marker of hydration status. Med Sci Sports Exerc. 2011;43(6):1080-4.

22. Neiva CM, Guerino MR, Mello MAR. Análise dos efeitos da desnutrição proteico-calórica sobre as respostas ao exercício agudo (single section) parâmetros metabólicos. Motriz. 1999;1(1):32-43.

23. McCaulley GO, Macbride JM, Cormie P, Hudson MB, Nuzzo JL, Quindry JC, et al. Acute hormonal and neuromuscular responses to hypertrophy, strength and power type resistance exercise. Eur J Appl Physiol. 2009;105(5):695-704.

24. Bradford MM. A rapid and sensitive method for the quantitation of microgram quantities of protein utilizing the principle of protein-dye binding. Analytical Biocheministry. 1976;72(1-2):248-54.

25. Hopkins WG, Marshall SW, Battherman AM, Hanin, J. Progressive statistics for studies in sports medicine and exercise science. Med Sci Sports Exerc. 2009;41(1):3-13.

26. Viveiros L, Costa EC, Moreira A, Nakamura FY, Aoki MS. Monitoramento do treinamento no judô: comparação entre a intensidade da carga planejada pelo técnico e a intensidade percebida pelo atleta. Rev Bras Med Esporte. 2011;17(4):266-69. 
27. Freitas VH, Miloski B, Bara Filho, MG. Quantificação da carga de treinamento através do método percepção subjetiva do esforço da sessão e desempenho no futsal. Rev Bras Cineantropom Desempenho Hum. 2012;14(1):73-82.

28. Smith DL, Shalmiyeva I, Deblois J, Winke M. Use of salivary osmolality to assess dehydration. Prehosp Emerg Care. 2012;16(1):128-35.

29. Walsh NP, Gleeson M, Pyne DB, Nieman DC, Dhabhar FS, Shephard RJ, et al. Position statement. Part two: maintaining immune health. Exerc Immunol Rev. 2011;17:64-103.

30. Muñoz CX, Johnson CE, Demartini JK, Huggins RA, McKenzie AL, Casa DJ, et al. Assessment of hydration biomarkers including salivary osmolality during passive and active dehydration. Eur J Clin Nutr. 2013;67(12):1257-63.

31. Smith DJ. A framework for understanding the training process leading to elite performance. Sports Med. 2003;33(15):1103-26.

32. Noakes TD. Fluid replacement during exercise. Exerc Sports Sci Rev. 1993;21:297-330.

33. Rossi L, Reis VAB, Azevedo COE. Desidratação e recomendaçôes para a reposição hídrica em crianças fisicamente ativas. Rev Paul Pediatr. 2010;28(3):337-45.

34. Walsh NP, Montague JC, Callow N, Rowlands AN. Saliva flow rate, total protein concentration and osmolality as potential markers of whole body hydration status during progressive acute dehydration in humans. Arch Oral Bio. 2004;49(2):149-54.

35. Karkoulias K, Habeos I, Charokopos N, Tsiamita M, Mazarakis A, et al. Hormonal responses to marathon running in nonelite athletes. Eur J Int Med. 2008;19(8):598-601.

36. Kraemer WJ, Ratamess NA. Hormonal responses and adaptations to resistance exercise and training. Sports Med. 2005;35(4):339-61.

37. Balsalobre-Fernádez C, Tejero-González CM, del Campo-Vecino J. Relationships between training load, salivary cortisol responses and performance during season training in middle and long distance runners. Plos One. 2014;9(8):1-6.

38. Tremblay MS, Copeland JL, Van Helder W. Influence of exercise duration on post-exercise steroid hormone responses in trained males. Eur J App Physiol. 2005;94(5-6):505-13.

39. Herman L, Foster C, Maher MA, Mikat RP, Porcari JP. Validity and reliability of the session RPE method for monitoring exercise training intensity. South Afr J Sports Med. 2006;18(1):14-7.

40. Borresen J, Lambert MI. Quantifying training load: a comparison of subjective and objective methods. Int J Sports Physiol Perform. 2008;3(1):16-30.

ENDEREÇO

Solange Marta Franzói de Moraes Universidade Estadual de Maringá Departamento de Ciências Fisiológicas Grupo de Estudos e Pesquisa em Fisiologia do Exercício Animal e Humana Avenida Colombo, 5790 - Jardim Universitário 87020-900 - Maringá - PR - Brasil email: smfmoraes@uem.br, smfmoraes@gmail.com
Submetido: 27/06/2015

Revisado: 13/10/2015

Aceito: 10/11/2015 
